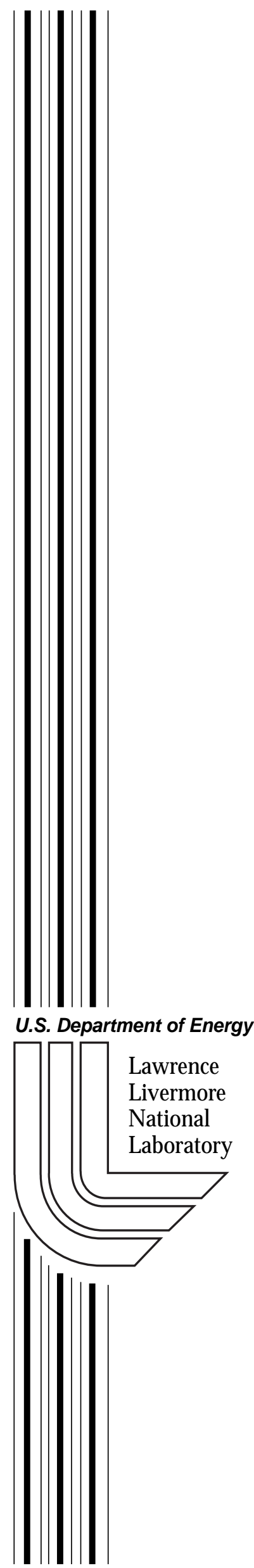

\title{
Microstructural Origins of Dynamic Fracture in Ductile Metals
}

J. Belak, J. Cazamias, M. Fivel, D. Haupt, J. Kinney, M. Kumar, R. Minich, R. Rudd, and A. Schwartz

February 17, 2004 


\section{DISCLAIMER}

This document was prepared as an account of work sponsored by an agency of the United States Government. Neither the United States Government nor the University of California nor any of their employees, makes any warranty, express or implied, or assumes any legal liability or responsibility for the accuracy, completeness, or usefulness of any information, apparatus, product, or process disclosed, or represents that its use would not infringe privately owned rights. Reference herein to any specific commercial product, process, or service by trade name, trademark, manufacturer, or otherwise, does not necessarily constitute or imply its endorsement, recommendation, or favoring by the United States Government or the University of California. The views and opinions of authors expressed herein do not necessarily state or reflect those of the United States Government or the University of California, and shall not be used for advertising or product endorsement purposes.

Work performed under the auspices of the U. S. Department of Energy by the University of California Lawrence Livermore National Laboratory under Contract W-7405-Eng-48. 


\title{
Microstructural Origins of Dynamic Fracture in Ductile Metals
}

J. Belak, J.U. Cazamias, M. Fivel, D. Haupt, J.H. Kinney, M. Kumar, R. Minich, R.E. Rudd, and

\author{
A. Schwartz \\ Lawrence Livermore National Laboratory, \\ University of Califonia, Livermore, CA 94550
}




\section{INTRODUCTION}

Dynamic fracture is a material failure process at high strain-rates. Here, we limit our discussion to spallation fracture during shock wave loading. When a compressive shock wave reflects from a free surface, internal states of tension are created. If this tension exceeds the rupture strength of the material, it fails by nucleating and growing microscopic voids in ductile metals and cracks in brittle solids. This effect, known as spallation, was reported by Hopkinson in 1872 [1]. Rinehart and Pierson [2] give an historical introduction to spallation and other aspects of high strain-rate deformation. This phenomenology of the nucleation and growth of microscopic voids is common to all fracture in ductile metals, including dynamic fracture.

The importance of pulse duration was not fully appreciated until the 1960's through the work of Butcher and colleagues [3, 4], leading to the concept of cumulative damage. This concept of damage accumulation was put on a strong experimental foundation by Barbee et al. [5], who performed gas gun recovery experiments and tediously measured the size and distribution of microscopic flaws using 2D microscopy. The resulting continuum material model of dynamic fracture is known as the SRI-NAG model $[6,7]$, for $\underline{\text { Nucleation }}$ And Growth. In a critical assessment of dynamic fracture, Meyers and Aimone [8] reviewed the abundant continuum spallation models and found the model of nucleation, growth, and coelescense of voids to qualitatively reproduce the observed extent of damage from spall recovery experiments. As in the work of Barbee et al. [5], Meyers and Aimone do not find any voids smaller than about $1 \mu \mathrm{m}$, suggesting that the 
voids either nucleated at that scale or when they are nucleated they rapidly grow to the micron size scale. The continuum models are phenomenological in nature with parameters fit to experiment. The advent of the Advanced Strategic Computing program has enabled direct numerical simulation of the nucleation and growth of microscopic voids. However, there exists no experimental data to both guide the modeling to the essential physical phenomena and to validate meso-scale modeling of dynamic fracture in ductile metals.

The goal of this project is to obtain this new experimental data for dynamic fracture in ductile metals by characterizing and analyzing the microscopic processes using modern experimental facilities not previously available. In particular, we measured with increased precision the damage produced during spallation fracture in ductile metals. Dynamic gas-gun recovery experiments were used to generate incipient spallation damage while concurrently measuring the free surface velocity wave profile. This wave profile has been used extensively to characterize spallation fracture. The concurrent measurement enables us to correlate the observed incipient damage with features in the wave profile, such as the velocity pull back. The incipient spallation damage was characterized using both advanced Synchrotron-based 3D X-Ray tomography and traditional 2D microscopy. A direct correlation was made between the traditional 2D microscopy and the 3D distribution of voids on the same sample. 2D microscopy, including optical, EBS/OIM, and TEM, was used to quantify, for the first time, the plastically deformed zone in the metal surrounding an incipiently grown void. 
Nanoindentation hardness experiments were used to quantify the increased strength in the plastically deformed zone from the greatly increased dislocation density.

\section{SCOPE OF WORK}

The microscopic flaws originate from material defects such as inclusions and grain boundaries. A microstructure-based predictive model of dynamic fracture must include the physics of these flaws. This project focuses on the origins of dynamic fracture in ductile metals, in particular the nucleation and growth of microscopic voids from material defects. Here, we recover incipiently damaged light ductile metals ( $\mathrm{Al}$, $\mathrm{Cu}, \mathrm{Va}, \mathrm{Ti})$ using the LLNL light gas gun and analyze the damage using both Synchrotron-based 3D X-Ray tomography and advanced 2D microscopy. These metals represent crystal structures of interest and are appropriate to future laser experiments. The tomography offers a unique window into the three dimensional structure of the incipient spall fracture plane and the experimental possibilities using NIF early light with multi-axis and time-resolved radiography.

This project establishes a paradigm that integrates several sequential experimental activities using modern facilities:

(1) Characterization and preparation of metals with known microstructure (single crystal, inclusion size $(\sim 0.1 \mu \mathrm{m})$ and distribution, and grain distribution). 
(2) Incipiently damaging and recovering a ductile metal subjected to a wellknown stress transient on the LLNL light gas gun. During FY01, this capability was established.

(3) Concurrent measurement of the free surface velocity wave profile (VISAR) during the spallation-recovery experiment. The motion of the free surface gives clues to the behavior of the material inside the sample.

(4) Characterization of incipiently damaged metals using Synchrotron-based 3D X-Ray tomography. The X-Ray tomography is a non-destructive technique which reveals details of the entire 3D incipient fracture surface. The experiments presented here were performed at the SSRL Synchrotron using 720 scan orientations. The very high photon flux of the Synchrotron enables a large number of scans during a short period of time.

(5) Characterization of incipiently damaged metals using modern 2D microscopy, including Electron Backscattering (EBS) methods and Transmission Electron Microscopy (TEM). The 2D microscopy reveals details of the interrelationship of void distribution with material microstructure and details of the plastic damage in the metal surrounding the incipiently grown voids.

(6) Nanoindentation hardness experiments within the plastically deformed zone surrounding incipiently grown voids. A very sharp diamond tip $(<0.1 \mu \mathrm{m}$ tip radius $)$ is poked into a carefully prepared surface while the effort required to produce an indentation is measured. The resulting mean 
pressure beneath the indenter is known as the surface hardness, which is related to the strength of the material.

All of the experiments are performed on the same sample and in many cases must be performed sequentially. For example, after the incipiently spalled sample is recovered, the sample is taken to the Synchrotron for the 3D tomography before being sectioned for the 2D microscopy. In this way we characterize the damage using both tomography and microscopy within the same region of material.

\section{TECHNICAL ACHIEVEMENTS}

This project focused on light metals to enable the Synchrotron-based 3D X-Ray tomography using available X-Ray energy (10's of keV). To illustrate the scope of the data, here we focus the discussion on aluminum, though we have applied the paradigm to copper and vanadium as well. Both polycrystal and single crystal samples were shocked to incipient spallation. The samples were 5-9's pure and the mean grain size in the polycrystalline samples was $200 \mu \mathrm{m}$. The samples were $5 \mathrm{~mm}$ thick and $25 \mathrm{~mm}$ in diameter with two additional "momentum" rings to control unwanted transverse momentum. The flyers were also aluminum to create a symmetric impact and varied in thickness from 1-3mm. The thickness of the flyer controls the pulse duration, i.e. the time at which the sample is under tension and the voids are allowed to nucleate and grow.

As a prerequisite to the project, we built and fielded a soft recovery system for the LLNL gas gun facility. Our design is based on the work of Rusty Gray from LANL [9]. 
The density of foam in the recovery tube is chosen so that the sample significantly embeds into the foam without impacting the rear surface of the tube. Key to our experimental recovery system is the concurrent measurement of the free surface velocity wave profile using VISAR interferometry. Here, we use a sacrificial mirror to deflect the light onto the sample and reflect the light back into the interferometer. After experimenting with several materials for the mirror, we chose a very thin glass slide that is coated with silver or gold. The rigidity of this sacrificial mirror was essential to obtaining a reproducible reflected light signal.

The resulting wave profiles for three shots from the same batch of polycrystalline aluminum are shown in Figure 1. The velocity of the impact is sufficient to create incipient spallation fracture. The initial pull-back velocity is comparable for all three shots and is related to the spall strength by $\sigma_{\mathrm{s}} \sim 1 / 2 \rho_{\mathrm{o}} \mathrm{c}_{\mathrm{o}} \Delta \mathrm{v}$. However, after the initial pull-back, there is significant difference in the wave profiles. This we interpret as due to the inhomogeneous nucleation and growth of porosity at the spall plane (see Figure 2). When the porosity links up to form a fracture surface, the wave is reflected, whereas, in regions of low porosity the wave is transmitted. Rather than monitoring the free surface at a single point, additional information about the inhomogeneous porosity nucleation and growth can be obtained in situ using a line imaging VISAR [10].

After the sample is recovered, we core a small $6 \mathrm{~mm}$ puck from the center of the sample, further minimizing edge effects. This puck is taken to the SSRL Synchrotron for 3D X-Ray tomographic analysis. The result for polycrystal sample \#932 is shown in Figure 3. This is the sample that generated the blue wave profile in Figure 1. A cylinder 
is drawn as a guide to the eye and reconstruction in the region of the spall plane is shown. We find a very inhomogeneous distribution of porosity with well separated regions of high and low porosity. The length scale from this data is about $400 \mu \mathrm{m}$, somewhat larger than the $200 \mu \mathrm{m}$ grain size in the material. This sample is then sectioned for 2D microscopy. The result is shown in Figure 3. The voids nucleate on the grain boundaries and grain boundary junctions. However, not all grain boundaries are active nucleation sites. This sample was scanned using Orientational Imaging Microscopy to reveal local lattice rotation. This is shown as the blue surrounding the voids in Figure 3. The tomography data can be filtered to directly compare to continuum spall models, for example, the porosity through the spall plane is shown in Figure 4. The additional peaks away from the main spall plane suggest correlated growth [11].

We have alse performed the experiments using 5-9's pure single crystal aluminum shocked along the [001], [011], [111], and [123] directions. Very little effect of shock direction was observed. The [123] direction was chosen to maximize the plasticity produced by the shock wave. The tomographic image for a sample shocked along the [011] direction is shown in Figure 5. Unlike the polycrystalline samples (Figure 2), here we find large well-separated voids, many of which are faceted. The void size distribution function is shown in Figure 6. The distribution is bimodal, i.e. there appears two populations of voids inside the sample: large voids of mean radius $\sim 30 \mu \mathrm{m}$ and small voids with an exponential distribution that scales to sizes smaller than the resolution of the tomography experiment $(3 \mu \mathrm{m})$. 
Shown in Figure 7 is an optical $2 \mathrm{D}$ microscope image from this single crystal aluminum sample. In preparation for the OIM experiment, the surface of the sectioned sample was carefully etched to remove damage created during the sectioning process. The result was a region of preferential etching surrounding each of the voids. This preferential etching occurs in regions of high dislocation density and was used in the first experiments to measure dislocation velocity. We have revealed, for the first time, the plastically deformed zone surrounding a dynamically grown void. The formation and evolution of this zone underlies every continuum physically-based model of spallation fracture.

To further quantify the mechanics of the material in this plastically deformed zone, we performed a series of nanoindentation hardness experiments across the plastic zone. The hardness is related to the local strength as $\mathrm{H} \sim 3 \mathrm{Y}$. The result is shown in Figure 8. We find an enhanced hardness within the zone which is consistent with higher dislocation content.

\section{SUMMARY}

The application of modern experimental diagnostics to the old problem of spallation fracture has provided new and interesting insights into the microscopic processes of void nucleation and growth. Synchrotron-based 3D X-Ray tomography was shown to directly reveal the full $3 \mathrm{D}$ structure of the incipient spall plane. The proof-of-principle experiments presented here were for light metals. In our future work we plan to extend this analysis to heavier metals using higher photon energies. The 
tomography data suggests that there are sub-micron voids in the recovered samples. These are difficult to observe using 2D microscopy because of the nature of the sample preparation. We are currently analyzing our samples using small angle scattering to extract the void distribution for void sizes between $1 \mathrm{~nm}$ and 1 micron. A further area where these results are guiding the underlying modeling of spallation is the analysis of the plastically deformed zone surrounding the incipiently grown voids.

\section{ACKNOWLEDGEMENTS}

This work was performed under the auspices of US Department of Energy by University of California-Lawrence Livermore National Laboratory (LLNL) under contract No. W-7405-Eng-48. 


\section{REFERENCES}

[1] Hopkinson, J., Proc. Manchester Lit. Soc., 11 (1872) 40.

[2] Rinehart, J.S., and Pearson, J., Behavior of Metals Under Impulsive Loads, ASME, Cleveland, OH, 1954 (reprinted by Dover, New York, NY, 1965); Rinehart, J.S., In Meyers, M.A., and Murr,L.E., (Eds.) Shock Waves and High-Strain-Rate Phenomena in Metals, Plenum, New York, NY, 1981, p3.

[3] Butcher, B.M., Barker, L.M., Munson, D.E., and Lundergan, C.D., AIAA J., 2 (1964) 977.

[4] Tuler, F.R., and Butcher, B.M., Int. J. Fract. Mech., 4 (1968) 431.

[5] Barbee Jr., T.W., Seaman, L., Crewdson, R., and Curran, D.R., J. Materals, 7 (1972) 393.

[6] Curran, D.R., Seaman, L., and Shockey, D.A., Phys. Today, 30 (1977) 46.

[7] Curran, D.R., Seaman, L., and Shockey, D.A., Phys. Rep., 147 (1987) 253.

[8] Meyers, M.A., and Aimone, C.T., Prog. Mater. Sci., 28 (1983) 1.

[9] Gray, G.T III, in High-Pressure Shock Compression of Solids, (Springer-Verlag, New York, 1993) 187.

[10] Chhabildas, L.C., Trott, W.M., Reinhart, W.D., Cogar, J.R., and Mann, G.A. , Proceedings of the 2001 APS Shock Compression of Condensed Matter (American Physical Society, New York, 2001) 483.

[11] Thissell, W.R., Zurek, A.K., Tonks, D.L., and Hixon, R.S., Proceedings of the 1999 APS Shock Compression of Condensed Matter (American Physical Society, New 
York, 2001) 451. 


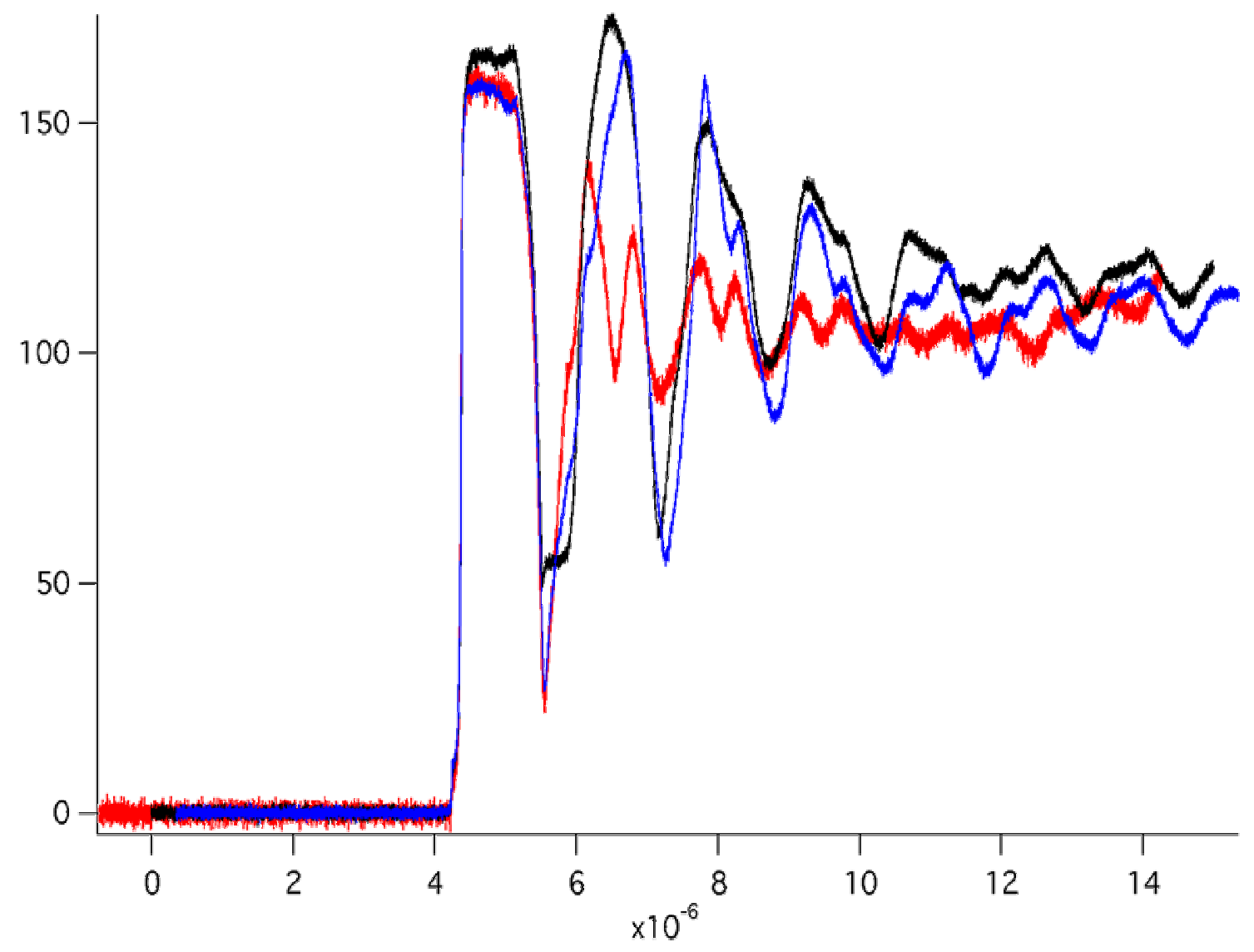

Figure 1. Three free surface velocity wave profiles for the same batch of 5-9's pure polycrystalline aluminum with 200 micron average grain size. The velocity $(\mathrm{m} / \mathrm{s})$ versus time (seconds). 


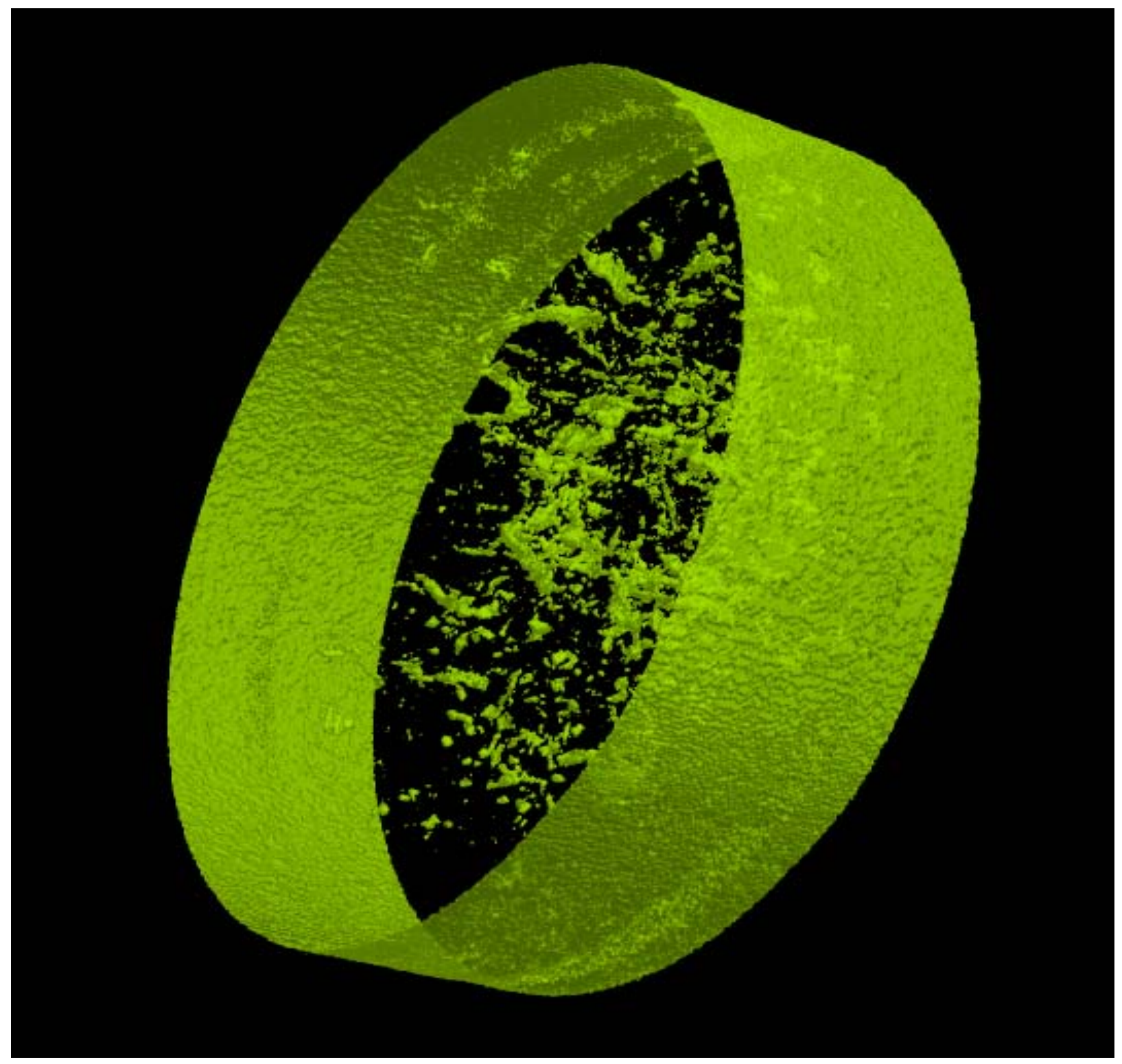

Figure 2. A 3D X-Ray tomographic image of incipient spallation fracture in polycrystalline aluminum. The radius of the cylinder is $6 \mathrm{~mm}$. This damage was produced by the stress trancient shown as the blue curve in Figure 1. 


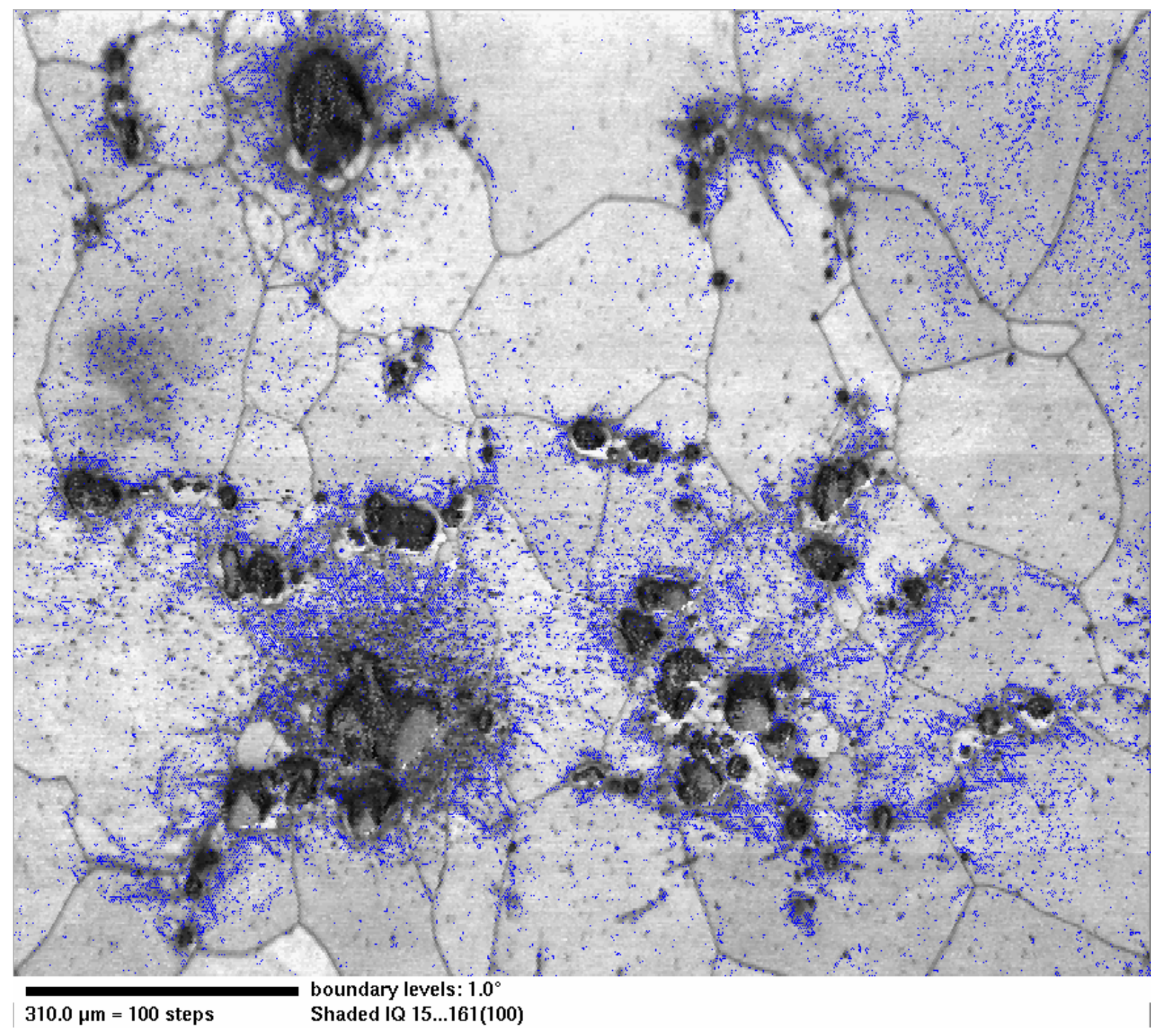

Figure 3. A 2D OIM image from the aluminum sample shown in Figure 2. Voids nucleate primarily on the grain boundaries and the blue region surrounding the voids shows local lattice rotation. 


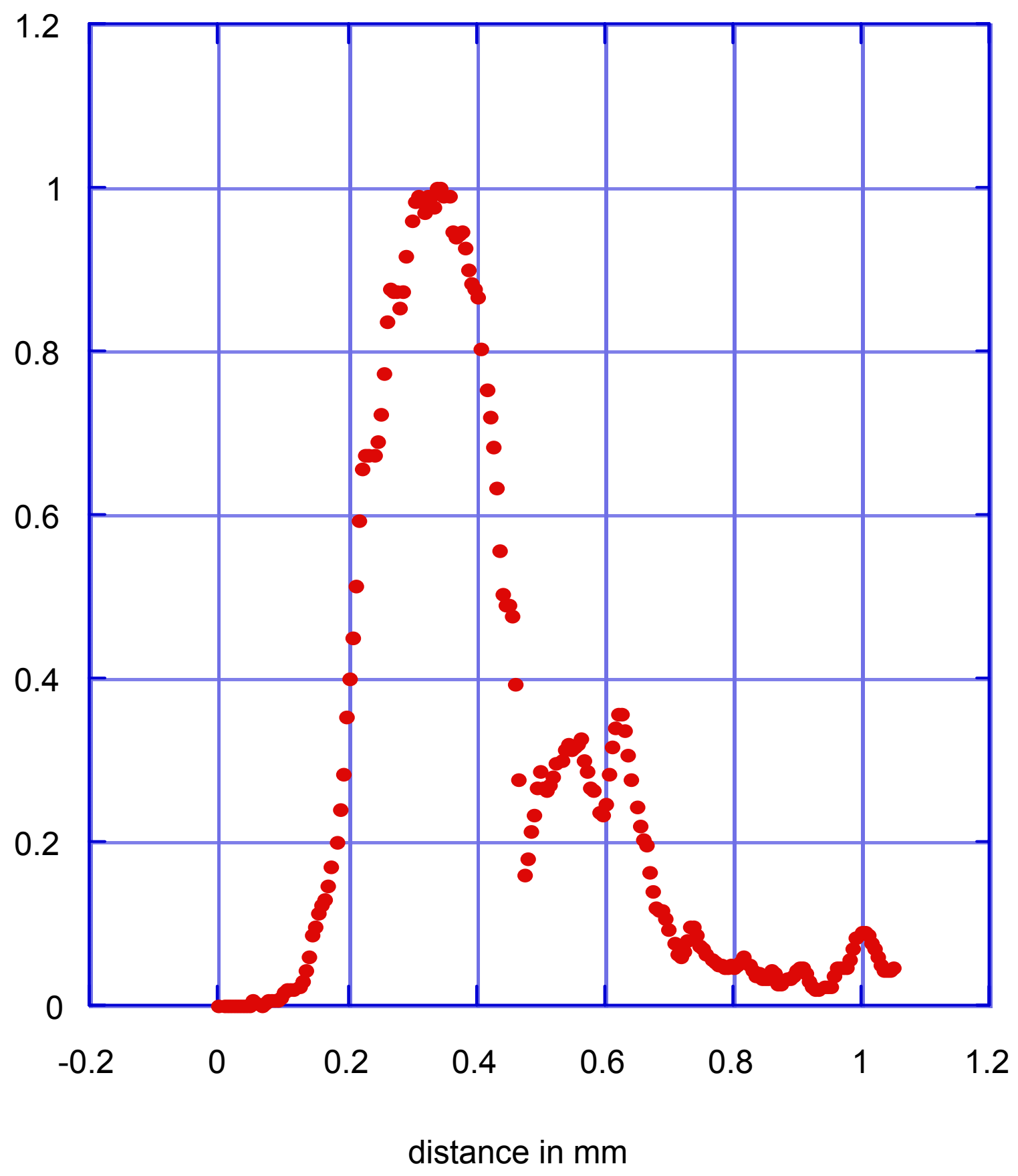

Figure 4. The porosity distribution through the spall plane as calculated for the polycrystalline tomography data shown in Figure 2. 


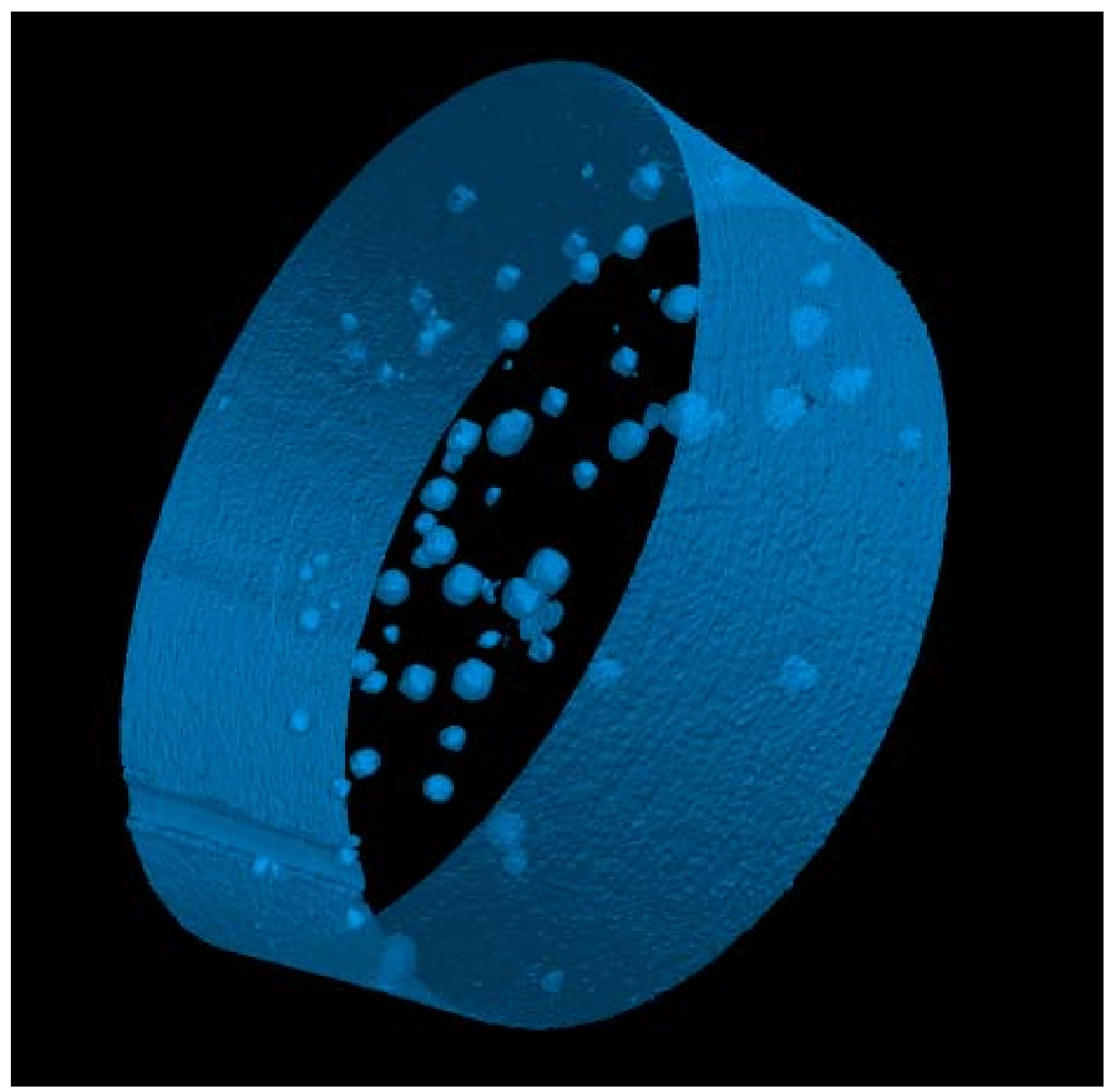

Figure 5. A 3D X-Ray tomographic image of incipient spallation fracture in 5-9's pure single crystal aluminum shocked along the [011] direction. The radius of the cylinder is $6 \mathrm{~mm}$. 


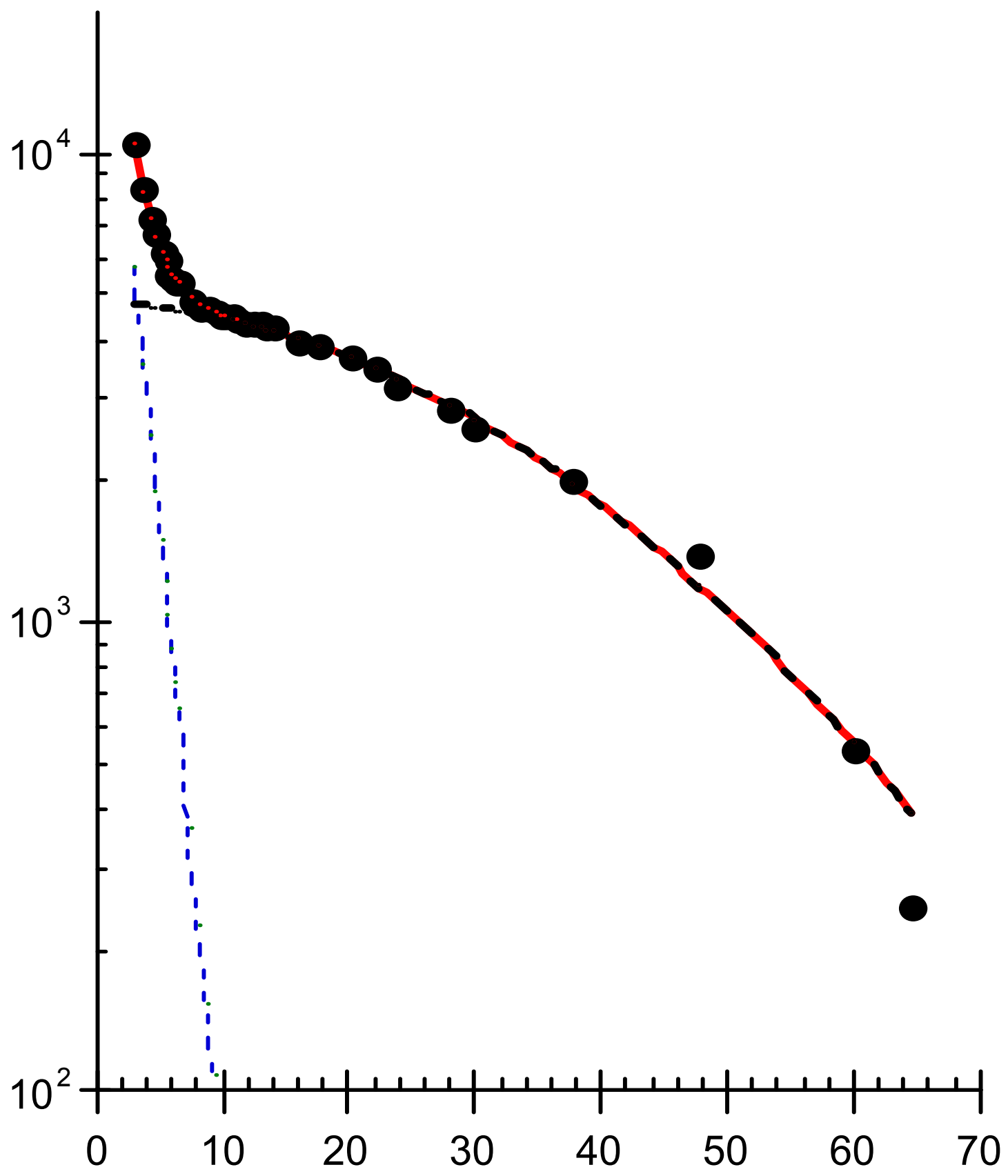

Figure 6. The void size distribution function for the single crystal data shown in Figure 5. (Radius in units of microns) 


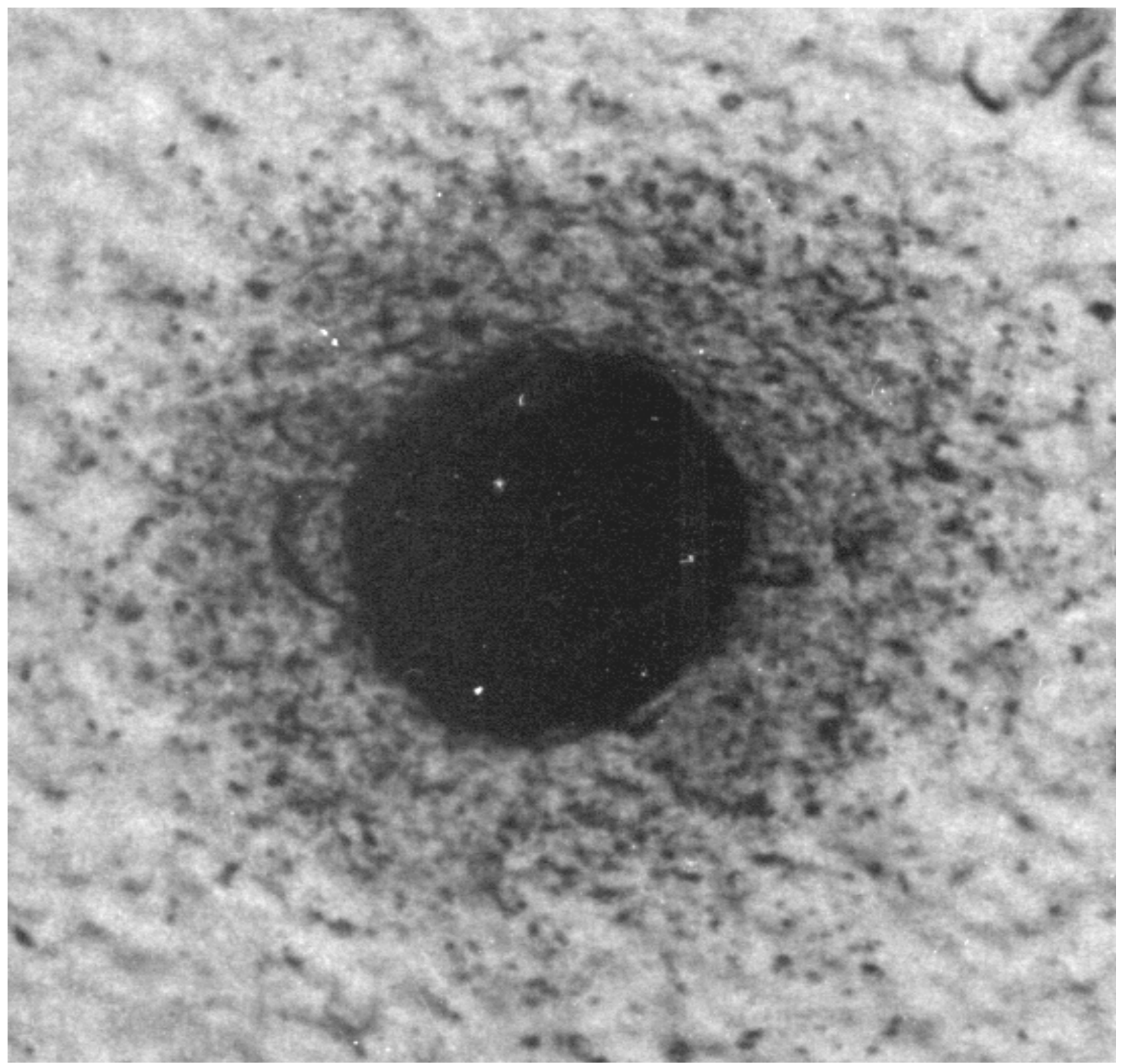

Figure 7. A 2D optical micrograph of the region surrounding one of the voids from the sample shown in Figure 5. The cloud surrounding the zone is revealed during the etching process and is associated with the plastically deformed zone. 


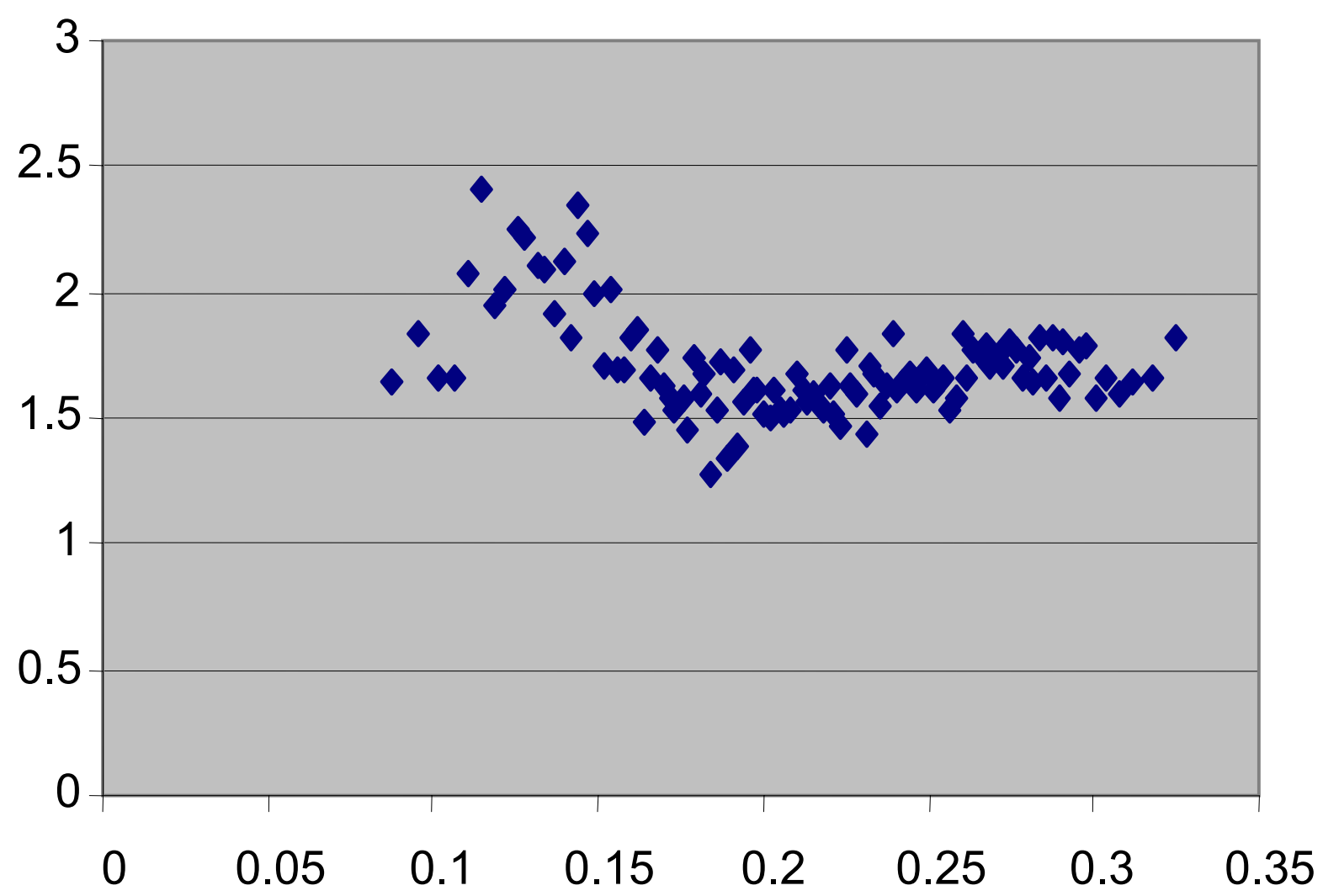

Figure 8. Nanoindentation hardness $(\mathrm{GPa})$ as a function of distance $(\mathrm{mm})$ from the center of one of the large voids shown in Figure 5. The enhanced hardness in the region surrounding the void indicates high dislocation content. 
